\title{
Orienting Towards Ensembles: From Single Cells to Neural Populations
}

\author{
Christopher M. Lewis ${ }^{1}$ and Andreea E. Lazar ${ }^{1,2,3}$ \\ ${ }^{1}$ Ernst Strüngmann Institute (ESI) for Neuroscience in Cooperation with Max Planck Society and ${ }^{2}$ Max Planck Institute for Brain Research, Frankfurt am \\ Main, 60528, Germany, and ${ }^{3}$ Frankfurt Institute for Advanced Studies, Frankfurt am Main, 60438, Germany \\ Review of Berens et al.
}

The tradition of single-cell electrophysiology has taught us a great deal about the response properties of individual cells in primary sensory cortices. However, the study of neurons in isolation provides a limited and possibly distorted picture of how the simultaneous activity of distributed neuronal populations is exploited by the brain to represent and respond to a variable environment in real time.

Single cells in primary visual cortex are sensitive to a range of stimulus properties: location, size, depth and, perhaps most famously, orientation (Hubel and Wiesel, 1962).

In a recent study, Berens et al. (2012) investigated orientation coding by populations of V1 neurons in alert macaque monkeys as the animals viewed static gratings of different orientation and contrast. They characterized the population response of up to 20 simultaneously recorded cells using a simple, neurally plausible decoder.

Critically, the decoder was invariant to stimulus contrast and could discriminate orientations using short integration intervals $(10-30 \mathrm{~ms})$ with only modest improvements for longer intervals (up to 70 $\mathrm{ms})$. In agreement with previous results in which odors were differentiated based on

Received Oct. 1, 2012; revised 0ct. 29, 2012; accepted Nov. 1, 2012.

Correspondence should be addressed to Christopher M. Lewis,

Deutschordenstraße 46, Frankfurt am Main, 60528, Germany. E-mail: chris.lewis@esi-frankfurt.de.

DOI:10.1523/JNEUROSCI.4658-12.2013

Copyright $\odot 2013$ the authors $\quad 0270-6474 / 13 / 330002-02 \$ 15.00 / 0$ population activity in the antenna lobe of locusts (Mazor and Laurent, 2005), Berens et al. (2012) show that stimulus orientation is best distinguished during the initial transient of the neural response, reaching peak performance after only 30-80 ms of stimulus presentation. These findings are of particular interest because they highlight the capacity for fast processing, enabling unambiguous identification or discrimination of visual information on timescales relevant for behavior.

Additionally, the sustained population responses to both static and moving gratings during continuous stimulation (500 ms) exhibited a high degree of time invariance. For example, a constant decoder trained on the spike count over the whole trial performed similarly to, although slightly worse than, an instantaneous decoder trained on short time bins (50 ms). Similar temporal invariance has been described by Nikolić et al. (2009), who recorded simultaneous responses from large populations of neurons in area 17 of anesthetized cats to briefly flashed visual stimuli (letters of the alphabet). In that study, the classification of the visual stimuli was similar when neuronal activity was integrated over short $(20 \mathrm{~ms})$ and long (100 ms) time intervals.

The study by Nikolić et al. (2009) is of particular interest because the investigators tested the performance of population decoders not only during the presentation of the current stimulus but also during subsequent blank periods. For the duration of this blank period (600 ms), in the absence of further stimulation, the population activity maintained information about the recently presented letter. Furthermore, when they investigated serial responses to sequences of letters, the evoked activity contained nearly equal information about both the current and the previous letter. These results highlight the ability of recurrent activity within primary visual cortex to maintain traces of recently flashed stimuli both in subsequent blank periods and concurrently with the presentation of new stimuli. Such persistent activity could be a powerful mechanism for integrating information sampled from continuous visual input, as required for the analysis of dynamic visual scenes.

Previous results using natural movies provide additional insight into this idea. Montemurro et al. (2008) found that considering spike times in relation to the phase of an ongoing low-frequency oscillation increased the information contained in population responses by $54 \%$ compared with spike counts alone. These findings indicate that network activity may provide more information about the sensory environment when taking into account the recent stimulus history as well as the intrinsic oscillatory dynamics in which they are embedded.

In contrast to recent results emphasizing the importance of correlated activity for coding of visual scenes at the level of 
large populations of retinal or cortical cells, Berens et al. (2012) found no meaningful performance increase by including (either explicitly or implicitly) the pairwise correlation structure of the population. They report that each unit can be treated as an independent encoder whose spike count follows the statistics of an independent Poisson distribution. This finding indicates that local effects within the network or across time are not essential for the discrimination of orientation. However, in a population of retinal ganglion cells, Pillow et al. (2008) found that excluding the contribution of pairwise correlations or history effects decreased their ability to reconstruct the stimulus by between $20 \%$ and $40 \%$. Further, Graf et al. (2011), also decoding V1 population responses (drifting gratings in anesthetized monkeys), showed that excluding the secondary statistics of pairwise correlation reduced their classification performance by $\sim 33 \%$ and increased their discrimination threshold (the difference in orientation necessary to distinguish between gratings).

These results may be attributable to the size and the relative densities of the populations used for decoding: 6-20 neurons spread across multiple cortical columns in the Berens et al. (2012) study, 27 contiguous cells in the retina in the Pillow et al. (2008) study, and 40-74 units also across multiple columns in the Graf et al. (2011) study. Additionally, both Pillow et al. (2008) and Graf et al. (2011) used timevarying stimuli, the resulting temporal dynamics of which may increase the importance of higher-order statistics. Last, in most of their analyses, Berens et al. (2012) used a long-duration static grating (500 ms), a stimulus known to induce considerable adaptation and to reduce the noise correlation of V1 cortical populations (Gutnisky and Dragoi, 2008).

The spontaneous spiking statistics of V1 populations has been shown to be exquisitely tuned during development to an ideal model of the visual environment (Berkes et al., 2011). Given that V1 neurons spontaneously discharge in patterns similar to those observed under stimulation, it seems likely that intrinsically driven correlated firing among sensory neurons reflects meaningful structure. To better understand the role that pairwise statistics play in encoding sensory stimuli it is important to investigate population coding under maximally naturalistic conditions.

Likewise, the immediate demands of task and cognitive state also frame and influence the response properties of cells in V1. It has been well established (Gilbert and Sigman, 2007) that dynamic topdown signals related to behavioral goals can have profound effects on both the response properties of individual cells, such as receptive field size and tuning, and the aggregate behavior of populations, such as attentional modulation of noise correlation or extra-classical suppression/facilitation. Such effects point to the importance of characterizing population activity under diverse behavioral conditions.

As has been stressed by Olshausen and Field (2005), our current understanding of computation in visual cortex has been built up piecemeal by single-unit studies and the arbitrary stimuli that elicit the maximal single-unit responses. However, in natural vision, the whole scene must be evaluated simultaneously, rather than via a constellation of uncoordinated feature extractors. Under such conditions the temporal and spatial interdependencies of scene structure directly constrain and inform the firing of distributed cell assemblies. As the current study by Berens et al. (2012) makes clear, understanding the rich dynamics of simultaneously recorded $\mathrm{V} 1$ cells, especially in alert animals, is an important step in the right direction. However, after establishing our methods in well characterized domains, such as orientation tuning, it will be important to apply them to explore novel territory.

The task ahead is made easier by new techniques such as multiphoton imaging and high-density multielectrode arrays. These technologies, combined with a pressing need to understand the function of the brain's integrated networks under natural conditions, call for the application of approaches like those used by Berens et al. (2012), combined with increasingly rich stimulation and behavioral protocols. Further, it is important to iteratively apply these techniques up the visual hierarchy to see how receiving areas integrate and further process the information passed on by earlier stages.

\section{References}

Berens P, Ecker AS, Cotton RJ, Ma WJ, Bethge M, Tolias AS (2012) A fast and simple population code for orientation in primate V1. J Neurosci 32:10618-10626. CrossRef Medline

Berkes P, Orbán G, Lengyel M, Fiser J (2011) Spontaneous cortical activity reveals hallmarks of an optimal internal model of the environment. Science 331:6013:83-87.

Gilbert CD, Sigman M (2007) Brain states: topdown influences in sensory processing. Neuron 54:677-696. CrossRef Medline

Graf ABA, Kohn A, Jazayeri M, Movshon JA (2011) Decoding the activity of neuronal populations in macaque primary visual cortex. Nat Neurosci 14:239-245. CrossRef Medline

Gutnisky DA, Dragoi V (2008) Adaptive coding of visual information in neural populations. Nature 452:220-224. CrossRef Medline

Hubel DH, Wiesel TN (1962) Receptive fields, binocular interaction and functional architecture in the cat's visual cortex. J Physiol 160: 106-154. Medline

Mazor O, Laurent G (2005) Transient dynamics versus fixed points in odor representations by locust antennal lobe projection neurons. Neuron 48:661-673. CrossRef Medline

Montemurro MA, Rasch MJ, Murayama Y, Logothetis NK, Panzeri S (2008) Phase-of-firing coding of natural visual stimuli in primary visual cortex. Curr Biol 18:375-380. CrossRef Medline

Nikolić D, Häusler S, Singer W, Maass W (2009) Distributed fading memory for stimulus properties in the primary visual cortex. PLoS Biol 7:e1000260. CrossRef Medline

Olshausen BA, Field DJ (2005) How close are we to understanding v1? Neural Comput 17: 1665-1699. CrossRef Medline

Pillow JW, Shlens J, Paninski L, Sher A, Litke AM, Chichilnisky EJ, Simoncelli EP (2008) Spatio-temporal correlations and visual signalling in a complete neuronal population. Nature 454:995-999. CrossRef Medline 